\title{
Efficacy of Conventional and Liposomal Povidone- Iodine in Infected Mesh Skin Grafts: An Exploratory Study
}

Peter M. Vogt · Joerg Hauser - Stefan Mueller - Bjoern Bosse •

Michael Hopp

Received: July 19, 2017 / Published online: October 10, 2017

(C) The Author(s) 2017. This article is an open access publication

\section{ABSTRACT}

Introduction: Infection is a major threat to wound healing and a leading cause of graft loss in patients undergoing meshed skin grafts (MSGs). Therefore, topical antisepsis is important in the overall treatment scheme.

Methods: An exploratory satellite group of 14 patients with infected MSGs were enrolled as part of a prospective, randomized, controlled, parallel-group, open-label, pilot Phase II study that investigated the efficacy and tolerability of 3\% liposomal povidone-iodine hydrogel (PVP-ILH, Repithel ${ }^{\circledR}$; RepiGel ${ }^{\circledR}$ ) versus chlorhexidine gauze in non-infected MSGs. The satellite group included both patients with infected wound beds prior to grafting and patients with infection of a previously placed graft, with MSG sizes ranging from 50 to $1000 \mathrm{~cm}^{2}$, who were randomized to treatment with (PVP-ILH) or $10 \%$ povidone-iodine ointment $\quad\left(\right.$ Betaisodona $^{\circledR} ; \quad$ BETADINE $\left.^{\circledR}\right)$.

Enhanced content To view enhanced content for this article go to http://www.medengine.com/Redeem/ 196CF0602C493233.

P. M. Vogt

Hannover Medical School, Hannover, Germany

J. Hauser

Alfried Krupp Hospital, Essen, Germany

S. Mueller $(\varangle) \cdot$ B. Bosse $\cdot$ M. Hopp

Mundipharma Research GmbH \& Co KG, Limburg,

Germany

e-mail: stefan.mueller@mundipharma-rd.eu
Medication was applied in a 2-mm layer and dressing changes with identical application of study medication took place daily. Wounds were evaluated by photoplanimetry, microbiologically and subjectively by patients and physicians.

Results: The results for the main study group have been reported previously. In the satellite group, both PVP-ILH and povidone-iodine ointment performed remarkably well with respect to lowering the bacterial count and restoring wound healing, with different emphasis. Povidone-iodine ointment showed excellent antibacterial efficacy with no detectable microorganisms by Day 10, and rapid re-epithelialization (mean $90 \%$ by Day 6). PVP-ILH also demonstrated rapid re-epithelialization (mean $72 \%$ by Day 6) with a trend towards improved subjective measures of wound healing quality. Four patients (40\%) receiving PVP-ILH experienced partial graft loss (10-15\% of total MSG area); no patients in the povidone-iodine ointment group experienced graft loss.

Conclusion: Our results suggest that povidone-iodine ointment has a strong role in managing infected wounds, especially when a high concentration of povidone-iodine may be warranted, while PVP-ILH indicated similar beneficial results on markers of wound healing quality in larger infected wounds.

Trial Registration: The trial was conducted prior to mandatory registration of drug 
products, PVP-ILH represents a medicated device in the EU and many other countries.

Funding: Mundipharma Research $\mathrm{GmbH} \& \mathrm{Co}$. KG.

Keywords: Liposomal povidone-iodine hydrogel; Mesh skin grafts; Microbial colonization; Povidone-iodine ointment; Pseudomonas aeruginosa; Staphylococcus aureus; Topical antisepsis; Wound healing; Wound infection

\section{INTRODUCTION}

Key goals of effective wound treatment are to keep the wound microorganism content low, stimulate the repair process, and enable optimized wound healing and quality of closure [1]. Some wounds of mixed etiology, e.g., burns and diabetic foot ulcers, are treated with meshed skin grafts (MSGs), which are a standard procedure for coverage of wounds because of their ease of harvest, applicability and resilience [2]. However, MSGs are very sensitive, and can be lost completely or partially if they do not grow properly into the underlying tissue [2]. Complete graft loss is the worst possible outcome. The grafting process must be repeated, reducing patient quality of life, increasing the workload for healthcare professionals, and increasing treatment costs. The loss of MSGs can be due to many factors with infection being one of the most prominent [2].

All wounds contain bacteria, but that does not mean that they are clinically infected [3-5]. There is a continuum in wound infection ranging from contamination to colonization and ultimately infection, with different necessities of medical intervention [5]. Understanding this sequence and using the right treatment is key to wound healing [5]. The presence of low counts of bacteria with no apparent negative effect on wound healing has also been described for MSGs [6].

Control of microbiological colonization and prevention of infection is vital and requires antimicrobial management and adequate wound dressings [7]. The worldwide change of pathogen characteristics, mainly induced by antibiotic overuse, is leading to a global increase of resistant pathogens. Therefore, antiseptic use is considered necessary to reserve antibiotics for indications where they are really needed [8].

Iodine has been used in wound treatment for more than 150 years. Povidone-iodine (PVP-I) has been popular since its discovery in 1955 because of its broad anti-microbial spectrum, versatility in wound treatment and lack of microbial resistance [4]. Furthermore, cell viability has been demonstrated at bactericidal concentrations of PVP-I in vitro [9], suggesting that it may not inhibit the wound healing process, unlike many other antiseptic solutions. The incorporation of PVP-I into liposomes was found to be very beneficial. PVP-I in a hydrogel base (PVP-ILH, hydrosome) combines the broad-spectrum antimicrobial activity of the antiseptic with the drug delivery and moisturizing properties of the liposomes and the hydrogel, thus presenting an innovative approach for wound healing $[1,3,10]$.

Paraffin gauze dressing is a standard skin graft dressing as it promotes more rapid and complete re-epithelization and is less painful than dry fine mesh gauze dressing [11]. Studies assessing non-infected skin grafts showed specific differences in wound healing depending on the antiseptic and dressing/formulation employed $[1,12]$. PVP-I ointment did not impede wound healing in a study of non-infected MSGs [6].

In some cases, it may be necessary to perform transplantation on already infected wounds (e.g., burn patients with fluid loss), or MSGs may become clinically infected subsequent to grafting, both of which will inhibit the speed and quality of wound healing and increase the risk of graft loss. Infected MSGs have not been assessed in clinical studies. The focus of this exploratory assessment is to investigate the efficacy and tolerability of PVP-ILH (Repithel ${ }^{\circledR}$; RepiGel $^{\circledR}$ ) and PVP-I ointment (Betaisodona ${ }^{\circledR}$; BETADINE $^{\circledR}$ ) in a "satellite group" of patients with infected MSGs.

\section{METHODS}

\section{Clinical Study}

Patients were enrolled into a prospective, randomized, open-label, active-controlled, 
parallel-group, pilot Phase II study, conducted between November 1997 and March 1999 at a single plastic surgery clinic in Germany. The study was conducted in accordance with $\mathrm{ICH}$ GCP, adhering to the ethical principles of the Helsinki Declaration of 1964, as revised in 1996. The Ethics Committee of the Landesaerztekammer Rheinland-Pfalz reviewed and approved the protocol, and written informed consent was obtained from all patients before enrollment.

Male and female patients aged over 18 years with meshed split-thickness skin grafts transplanted to various full-thickness wounds were enrolled after written consent. Specifically excluded were patients with alcohol, medication or drug abuse, hyperthyreosis or other thyroid disease, and dermatitis herpetiformis during or 4 weeks before or after radioiodine therapy or before nuclear medical diagnostics. Patients with non-infected MSGs were randomized to treatment with 3\% PVP-ILH or chlorhexidine gauze as described previously [3], and are subsequently not discussed here. An exploratory satellite group of patients with infected MSGs were randomized to treatment with 3\% PVP-ILH or 10\% PVP-I ointment and are the focus of this paper. Patients were randomized using blocking methodology according to patient number, allocated sequentially as patients were enrolled into the study.

Infection was based on clinical assessment of the wound/graft and classical signs of infection (redness, heat, swelling, pain) and more specifically in wound healing on what was then understood to be critical colonization and the symptoms summarized as NERDS (including, e.g., non-healing and excessive exudation). Since the inclusion of patients with infected MSGs was a small exploratory part of the study, there were no specific inclusion criteria regarding the type or size of infection, and the group was heterogenous in nature. Some patients already had clinically frank wound-bed infections at the time of grafting, and the grafting day coincided with the start of treatment due to medical reasons. In other patients, the infection occurred at different intervals (up to 4 days) after grafting and the start of the healing process.
Application of study medication started immediately following diagnosis of the skin graft infection. In both treatment groups, study medication was applied in a $2-\mathrm{mm}$ continuous layer. A paraffin gauze and a tie-over dressing were placed on top. Dressing changes with identical application of study medication took place daily until the next operation (or healing, if applicable). Patients were followed-up until wound closure, which occurred by Day 12 for all patients.

\section{Measurement of Epithelialization and Microbiological Assessment}

The epithelialization of the mesh holes on skin grafts was assessed by photoplanimetry (standardized photos, observer-blind differentiation by computer-aided measurements between epithelialized and non-epithelialized surface areas defined by colorization and the calculation of re-epithelialization per time), which was performed every 2 days as described previously $[3,12]$. Microbiological assessment was performed on Days 0, 4 and 10 by swab sample with qualitative determination of the microorganisms present.

\section{Physician and Patient Assessments}

The physician performed a clinical assessment of infected MSGs including presence/absence of secretion, redness, coatings, discoloration and brown iodine stain, and subjective assessment of the degree of moisture every 2 days. On the same days, the patient assessed wound treatment via multiple choice questions about wound pain, itch and dressing change. The healing process was evaluated subjectively by the treating physician (antiseptic efficacy, ease of handling, wound healing quality, elasticity of skin grafts, suppleness and appearance) at the end of treatment. Safety was assessed by the monitoring and reporting of adverse events. Measurements of thyroid hormones triiodothyronine $\left(\mathrm{T}_{3}\right)$, thyroxone $\left(\mathrm{T}_{4}\right)$ and thyroid-stimulating hormone (TSH) were performed to evaluate local and systemic tolerability. 


\section{Statistical Analysis}

Data evaluation was descriptive and exploratory. Exploratory $p$ values for the treatment group comparison were calculated using Fisher's exact test (analyses of physician and patient assessments) and the Mann-Whitney $U$ test (analyses of re-epithelialization and graft failure).

\section{RESULTS}

\section{Patient Characteristics}

A total of 14 patients with infected MSGs were enrolled in the satellite group. Ten patients were randomized to 3\% PVP-ILH and 4 patients were randomized to $10 \%$ PVP-I ointment. The PVP-ILH group included 5 men and 5 women aged 23-68 years and the PVP-I ointment group included 3 men and 1 woman aged 38-70 years (Table 1). The main reason for MSG was burns and the mean MSG size was $450 \mathrm{~cm}^{2}$ for the PVP-ILH group and $270 \mathrm{~cm}^{2}$ for the PVP-I ointment group. The mean duration of treatment was 9.0 days (range 5-11 days) for PVP-ILH and 6.8 days (range 5-9 days) for
PVP-I ointment. All 14 patients in the satellite group completed the study (wound closure) and were included in efficacy and safety analyses.

\section{Efficacy}

Overall, both treatments were well tolerated with no statistically significant differences in healing efficacy. By Day 6, the mean re-epithelialized area was $90 \%$ for PVP-I ointment and $72 \%$ for PVP-ILH (Fig. 1). By Day 12, the mean re-epithelialized areas were $100 \%$ for PVP-I ointment and 99\% for PVP-ILH. A similar healing trend was observed when considering only the 9 patients whose infection started on Day 0 (data not shown). The photoplanimetry results are supported by the physician's assessment of the tendency for wound healing, which for PVP-I ointment was rated as "good" in 14/15 (93\%) of assessments and "moderate" in 1/15 (7\%) of assessments, and for PVP-ILH was rated as "very good" in $1 / 49(2 \%)$ of assessments, "good" in $36 / 49$ (73\%) of assessments and "moderate" in 12/49 (25\%) of assessments.

There were no notable differences between the two treatment groups in the physician's assessment of secretion, redness, coatings,

Table 1 Patient demographic and baseline characteristics

\begin{tabular}{llll}
\hline Parameter & Statistic & $\begin{array}{l}\text { Liposomal PVP-I hydrogel } \\
(\boldsymbol{N = 1 0 )}\end{array}$ & $\begin{array}{l}\text { PVP-I ointment } \\
(\boldsymbol{N}=\mathbf{4})\end{array}$ \\
\hline Gender $[n(\%)]$ & Male & $5(50)$ & $3(75)$ \\
& Female & $5(50)$ & $1(25)$ \\
Age (years) & Mean & 40.8 & 52.2 \\
& Range & $23-68$ & $38-70$ \\
Transplantation reason & Burns & $6(60)$ & $2(50)$ \\
{$[n(\%)]$} & Trauma, flap donor & $2(20)$ & $1(25)$ \\
& site & $2(20)$ & $1(25)$ \\
& Other & 450 & 270 \\
Transplantation size $\left(\mathrm{cm}^{2}\right)$ & Mean & $50-1000$ & $100-450$ \\
\hline
\end{tabular}

$n$ (\%) number and \% of patients, $N$ number of patients in treatment group 


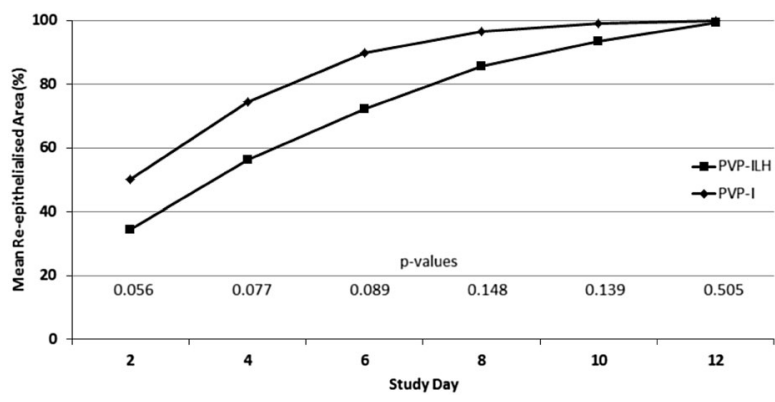

Fig. 1 Mean re-epithelialized area (\%) during the study (with exploratory $p$ values). Number of patients was 10 for PVP-I ointment ( $n=8$ for study Day 12 ) and 4 for PVP-ILH

discoloration, brown iodine stain, and moisture (Table 2). The grafts were typically rated as "moist" or "reasonably moist" in both treatment groups; dry grafts occurred only in isolation, in most cases towards the end of the study. The subjective assessment of wound healing by the physician showed a trend for better antiseptic efficacy and appearance with PVP-I ointment, while results for ease of handling, wound healing quality, elasticity and suppleness appeared to favor PVP-ILH. However, there was no statistically significant difference between the two treatments for any of these parameters (Table 3).

Overall, ten different pathogens were found in the MSG wounds, with the most prevalent being S.aureus. Patients in both treatment groups tested positive up to Day 4; however, by Day 10, the microbial count was 0 in the PVP-I ointment group, while patients still tested positive for $S$. aureus, P. miribalis, E. faecalis, $P$. aeruginosa and E. coli at Day 10 in the PVP-ILH group (Table 4). Graft failure occurred in four patients (40\%) who received PVP-ILH, but graft loss was only partial and involved small areas (10-15\% of total MSG). None of the four patients in the PVP-I ointment group experienced graft loss and the treatment comparison for graft loss was statistically significant $(p=0.0222)$.

The overall assessment of wound treatment by patients in terms of wound pain, itching, and comfort of dressing change was similar for PVP-ILH and PVP-I (Table 5).

\section{Safety}

Both topically applied treatments were well tolerated. No serious adverse events occurred and thyroid function parameters were within normal limits, showing no systemic effects (data not shown).

\section{DISCUSSION}

Antiseptics in wound care are to control the amount and the type of bacteria in a wound. MSGs are very sensitive with almost a quarter lost, mainly due to infection [2]. Therefore, the presence of excessive amounts of bacteria in open skin wounds is a major challenge to graft take and wound healing [3]. Few studies have been published on how to treat clinically infected MSGs. Consequently, part of the present study was performed to assess the efficacy of PVP-ILH versus PVP-I in patients with infected MSGs.

MSGs can easily be analysed objectively, e.g., by photometric assessment, and subjectively by health care professionals and patients. However, in some cases, such as burn wounds covering large total body surface areas (TBSAs) and chronic wounds, the results must be interpreted with caution, as these may represent more complex wound healing settings.

In our study, both PVP-ILH and PVP-I ointment performed remarkably well towards lowering the bacterial burden in infected MSG wounds; thus, enabling healing. PVP-I ointment showed excellent antibacterial efficacy with no detectable microorganisms by Day 10, and rapid wound healing (mean 90\% re-epithelialization by Day 6). PVP-ILH also demonstrated impressive wound healing capability (mean $72 \%$ re-epithelialization by Day 6), especially considering the initial wound size (Table 1), with a trend towards improved subjective measures of wound healing quality. The greater antibacterial efficacy observed with PVP-I ointment is likely to be due to the higher iodine content (10\%) compared with PVP-ILH (3\%), which may become relevant in the case of a massive infection. The antimicrobial effect of PVP-I enabling wound healing is probably 
Table 2 Subjective assessment of infected mesh skin grafts across all visits

\begin{tabular}{|c|c|c|c|}
\hline Parameter & Assessment & $\begin{array}{l}\text { Liposomal PVP-I hydrogel } \\
(N=10)\end{array}$ & $\begin{array}{l}\text { PVP-I ointment } \\
(N=4)\end{array}$ \\
\hline \multicolumn{2}{|c|}{ Total number of clinical assessments } & 60 & 19 \\
\hline \multirow[t]{3}{*}{ Secretion $[n(\%)]$} & Yes & $19(32)$ & $9(47)$ \\
\hline & No & $41(68)$ & $10(53)$ \\
\hline & Exploratory $p$ value & 0.273 & \\
\hline \multirow[t]{3}{*}{ Redness $[n(\%)]$} & Yes & $39(65)$ & $10(53)$ \\
\hline & No & $21(35)$ & $9(47)$ \\
\hline & Exploratory $p$ value & 0.209 & \\
\hline \multirow[t]{3}{*}{ Coatings $[n(\%)]$} & Yes & $6(10)$ & $3(16)$ \\
\hline & No & $54(90)$ & $16(84)$ \\
\hline & Exploratory $p$ value & 0.443 & \\
\hline \multirow[t]{3}{*}{ Discoloration $[n(\%)]$} & Yes & $1(2)$ & $0(0)$ \\
\hline & No & $59(98)$ & $19(100)$ \\
\hline & Exploratory $p$ value & 0.999 & \\
\hline \multirow[t]{4}{*}{ Brown iodine stain $[n(\%)]$} & No data & $9(15)$ & $0(0)$ \\
\hline & Yes & $0(0)$ & $1(7)$ \\
\hline & No & $51(85)$ & $14(93)$ \\
\hline & Exploratory $p$ value & 0.227 & \\
\hline \multirow[t]{5}{*}{ Moisture $[n(\%)]$} & Moist & $10(20)$ & $4(27)$ \\
\hline & Reasonably moist & $32(64)$ & $9(60)$ \\
\hline & Dry & $8(16)$ & $2(13)$ \\
\hline & Very dry & $0(0)$ & $0(0)$ \\
\hline & Exploratory $p$ value & 0.911 & \\
\hline
\end{tabular}

$n$ (\%) absolute number and \% of all assessments, $N$ number of patients in treatment group

further supported by the liposomal hydrogel in the PVP-ILH group. The high efficacy of PVP-I ointment against a broad spectrum of bacteria over the 10-day course of our study reflects the results of previous reports [6].

PVP-I has been compared to other antiseptics and has performed remarkably well [13]. This was highlighted in published studies in various non-infected skin grafts (mainly donor sites), that represent frequently used model wounds.
Chlorhexidine dressing, despite being highly effective against a wide range of Gram-positive and Gram-negative bacteria, does not perform as well as PVP-ILH in terms of re-epithelialization rate and wound healing [1], and has additional disadvantages, such as adhesion to wound surfaces and pain from the irritation caused by this dressing [14]. Octenidine dihydrochloride has significantly lowered bacterial count of skin graft donor sites but has failed to reduce the time to wound closure versus inert dressing (with a trend for inert dressing) [15]. 
Table 3 Results of wound healing assessment by the physician

\begin{tabular}{|c|c|c|c|}
\hline Parameter & Degree & $\begin{array}{l}\text { Liposomal PVP-I hydrogel } \\
(N=10)\end{array}$ & $\begin{array}{l}\text { PVP-I ointment } \\
(N=4)\end{array}$ \\
\hline \multirow[t]{4}{*}{ Antiseptic efficacy $[n(\%)]$} & Very good & $0(0)$ & $1(25)$ \\
\hline & Good & $8(80)$ & $3(75)$ \\
\hline & Moderate & $2(20)$ & $0(0)$ \\
\hline & Exploratory $p$ value & 0.341 & \\
\hline \multirow[t]{3}{*}{ Ease of handling $[n(\%)]$} & Very good & $3(30)$ & $0(0)$ \\
\hline & Good & $7(70)$ & $4(100)$ \\
\hline & Exploratory $p$ value & 0.505 & \\
\hline \multirow[t]{4}{*}{ wound healing quality $[n(\%)]$} & Good & $6(60)$ & $2(50)$ \\
\hline & Somewhat good & $2(20)$ & $2(50)$ \\
\hline & Moderate & $2(20)$ & $0(0)$ \\
\hline & Exploratory $p$ value & 0.510 & \\
\hline \multirow[t]{4}{*}{ Elasticity $[n(\%)]$} & Good & $6(60)$ & $1(25)$ \\
\hline & Somewhat good & $2(20)$ & $1(25)$ \\
\hline & Moderate & $2(20)$ & $2(50)$ \\
\hline & Exploratory $p$ value & 0.608 & \\
\hline \multirow[t]{4}{*}{ Suppleness $[n(\%)]$} & Good & $6(60)$ & $1(25)$ \\
\hline & Somewhat good & $1(10)$ & $1(25)$ \\
\hline & Moderate & $3(30)$ & $2(50)$ \\
\hline & Exploratory $p$ value & 0.406 & \\
\hline \multirow[t]{5}{*}{ Appearance $[n(\%)]$} & Very good & $2(20)$ & $1(25)$ \\
\hline & Good & $4(40)$ & $3(75)$ \\
\hline & Somewhat good & $3(30)$ & $0(0)$ \\
\hline & Moderate & $1(10)$ & $0(0)$ \\
\hline & Exploratory $p$ value & 0.811 & \\
\hline
\end{tabular}

Results were assessed as very good, good, somewhat good, moderate, somewhat poor, poor, very poor $n(\%)$ number (\%) of patients in assessment category, $N$ number of patients in treatment group

Silver dressings also do not differentiate against established treatment on a clinical level (time to wound closure, graft loss) [16], and have been shown to be poorly tolerated by skin graft patients in terms of pain scores [17]. A recent randomized, double-blind, controlled study in full-thickness grafts showed no effect of polyhexanide-soaked dressings on postoperative bacterial loads and increased risk of surgical site infections compared with inert dressings [18]. In contrast, PVP-I has shown a highly bactericidal effect with earlier onset of healing than inert dressing in MSG recipient sites [6]. This makes the results of enhanced wound healing in MSG recipient sites, both against chlorhexidine gauze and inert paraffin gauze, 
Table 4 Microorganisms identified in infected mesh skin grafts

\begin{tabular}{|c|c|c|c|c|c|c|}
\hline \multirow[t]{2}{*}{ Microorganism } & \multicolumn{3}{|c|}{ Liposomal PVP-I hydrogel $(N=10)$} & \multicolumn{3}{|c|}{ PVP-I ointment $(N=4)$} \\
\hline & Day 0 & Day 4 & Day 10 & Day 0 & Day 4 & Day 10 \\
\hline Staphylococcus epidermidis & - & 2 & - & - & - & - \\
\hline Staphylococcus capitis & 1 & - & - & - & - & - \\
\hline Staphylococcus aureus & 6 & 5 & 4 & 4 & 3 & - \\
\hline Enterococcus faecalis & - & 2 & 1 & - & - & - \\
\hline Pseudomonas stutzeri & - & 1 & - & - & - & - \\
\hline Pseudomonas aeruginosa & 2 & 1 & 1 & 1 & 1 & - \\
\hline Escherichia coli & 4 & 2 & 1 & - & - & - \\
\hline Proteus mirabilis & 2 & 3 & 3 & - & - & - \\
\hline Acinetobacter anitratus & 2 & 1 & - & - & - & - \\
\hline Streptococcus faecalis & 2 & - & - & - & - & - \\
\hline Total & 19 & 17 & 10 & 5 & 5 & 0 \\
\hline
\end{tabular}

$N$ number of patients in treatment group

noteworthy. It should be considered that not only the antiseptic but also the dressing contributes to a good overall result [14].

No delay in wound healing time of $10 \%$ PVP-I ointment (compared with simple vaseline gauze) has been found in split skin graft patients [6], while 3\% PVP-ILH even enhanced wound healing in non-infected MSGs compared to chlorhexidine and simple paraffin gauze [3, 12]. Slightly accelerated wound healing, no infection and good local tolerance has been observed in an early study in donor sites for PVP-I solution versus dry and moist cotton gauze as well as two other antiseptics [19]. This small, early study also demonstrated the advantage of moist wound healing. Overall, these observations are in agreement with recent in vitro results showing lower cytotoxicity of PVP-I compared to other antiseptics [9], and reviews of iodine in wound care concluding that PVP-I does not impair wound healing $[4,13]$.

Our data in infected MSGs extend these findings, with both PVP-I ointment and PVP-ILH showing fast re-epithelialization, with favorable assessments of healing by the treating physician. Consistent with other reports $[6,13]$, we found no evidence of an effect of PVP-I ointment (or PVP-ILH) on healthy thyroid function.

As detailed in the "Introduction", the issue of transplant loss during treatment is of particular clinical relevance. None of the four patients with infected MSGs who received PVP-I ointment in our study experienced graft loss, which can likely be attributed to the pronounced antimicrobial efficacy of the PVP-I ointment. Partial graft loss involving small areas occurred in four patients (40\%) in the PVP-ILH group; however, it must be considered that our study population already had clinically frank graft infections at the time of treatment. In the previously reported group of non-infected MSGs, only $5 \%$ of patients treated with PVP-ILH suffered transplant loss [1].

The results of this study have their limitations because of the low number of patients with infected MSGs included in this satellite group and the treatment group disparity; in total, 10 patients received PVP-ILH and 4 patients received PVP-I ointment. As this was only a satellite group of patients with infected MSGs, recruitment was terminated when the 
Table 5 Patient assessment of wound pain, itch and comfort of dressing change

\begin{tabular}{|c|c|c|c|}
\hline Parameter & Characteristics & $\begin{array}{l}\text { Liposomal PVP-I hydrogel } \\
(N=10)\end{array}$ & $\begin{array}{l}\text { PVP-I ointment } \\
(N=4)\end{array}$ \\
\hline \multicolumn{2}{|c|}{ Total number of patient assessments } & 100 & 100 \\
\hline \multirow[t]{6}{*}{ Wound Pain $[n(\%)]$} & None & $16(32)$ & $2(13)$ \\
\hline & Mild & $16(32)$ & $7(47)$ \\
\hline & Moderate & $11(22)$ & $6(40)$ \\
\hline & Severe & $7(14)$ & 0 \\
\hline & Unbearable & 0 & 0 \\
\hline & Exploratory $p$ value & 0.146 & \\
\hline \multirow[t]{6}{*}{ Itch $[n(\%)]$} & None & $49(98)$ & $12(80)$ \\
\hline & Mild & $1(2)$ & $3(20)$ \\
\hline & Moderate & 0 & 0 \\
\hline & Severe & 0 & 0 \\
\hline & Unbearable & 0 & 0 \\
\hline & Exploratory $p$ value & 0.036 & \\
\hline \multirow[t]{5}{*}{ Dressing change $[n(\%)]$} & Uncomfortable & $42(84)$ & $10(67)$ \\
\hline & $\begin{array}{l}\text { Moderately } \\
\text { uncomfortable }\end{array}$ & $7(14)$ & $4(26)$ \\
\hline & Very uncomfortable & $1(2)$ & $1(7)$ \\
\hline & Extremely uncomfortably & 0 & 0 \\
\hline & Exploratory $p$ value & 0.204 & \\
\hline
\end{tabular}

$n(\%)$ absolute number and \% of all assessments, $N$ number of patients in treatment group

main part of the study in patients with non-infected MSGs was completed, which, combined with the blocking method of randomization used, resulted in treatment group imbalance. The interpretation and applicability of the results is also confounded by the heterogeneity of the satellite group, attributable to the different time points at which infection had occurred in relation to the time of grafting. This made patients difficult to compare in terms of the main criterion of photoplanimetry because a certain degree of re-epithelialization may have taken place before treatment started, meaning that the baseline point did not lie uniformly at Day 0 . While the photoplanimetry assessments were objective, assessments of wound healing by the physician and patient were subjective and unvalidated. However, no validated metrics exist for assessing infected MSGs, and our methods were intended to give at least an indication of the success of the procedure, while subjective assessment adds valuable information not easily obtainable from objective parameters. The $p$ values obtained from the statistical analyses can only be considered exploratory due to the small sample size in this satellite group of patients with infected MGSs.

Future studies must therefore focus on recruiting more eligible patients to better 
elucidate the effects of PVP-ILH and PVP-I in infected MSGs. Additional microbial assessments (e.g., on Days 6 and 8) could help better understand the removal kinetics of prevalent infecting bacteria. This may generate valuable information for health care practitioners and give an insight into existing clinical data.

\section{CONCLUSION}

In conclusion, our results in infected MSGs show a trend for more effective bacterial removal and more rapid re-epithelialization (by physician assessment and photoplanimetry) with 10\% PVP-I ointment, which may be the initial treatment of choice for infected MSGs. Liposomal 3\% PVP-I hydrogel achieved better scores in subjective assessments of quality of wound healing, and may be preferred as a dressing for non-infected MSGs.

\section{ACKNOWLEDGEMENTS}

Sponsorship for this study and article processing charges were funded by Mundipharma Research $\mathrm{GmbH} \&$ Co.KG. Medical writing support and materials were funded by Mundipharma Research GmbH \& Co.KG. All authors had full access to all of the data in this study and take complete responsibility for the integrity of the data and accuracy of the data analysis.

All named authors meet the International Committee of Medical Journal Editors (ICMJE) criteria for authorship for this manuscript, take responsibility for the integrity of the work as a whole, and have given final approval to the version to be published. Editorial assistance in the preparation of this manuscript was provided by Karen Mower of Scientific Editorial. Support for this assistance was funded by Mundipharma Research GmbH \& Co.KG.

Disclosures. Peter Vogt and Joerg Hauser have nothing to disclose. Stefan Mueller is an employee of Mundipharma Research $\mathrm{GmbH} \&$ Co.KG. Bjoern Bosse is an employee of
Mundipharma Research GmbH \& Co.KG. Michael Hopp is an employee of Mundipharma Research GmbH \& Co.KG.

Compliance with Ethics Guidelines. All procedures followed were in accordance with the ethical standards of the responsible committee on human experimentation (institutional and national) and with the Helsinki Declaration of 1964, as revised in 1996. Informed consent was obtained from all patients for being included in the study. These results have previously been presented in a poster presentation at the 16th European Burns Association (EBA) Congress, 16-19 September 2015, Hannover, Germany.

Trademarks. ®) REPITHEL, REPIGEL, BETAISODONA and BETADINE are Registered Trademarks.

Open Access. This article is distributed under the terms of the Creative Commons Attribution-NonCommercial 4.0 International License (http://creativecommons.org/licenses/ by-nc/4.0/), which permits any noncommercial use, distribution, and reproduction in any medium, provided you give appropriate credit to the original author(s) and the source, provide a link to the Creative Commons license, and indicate if changes were made.

\section{REFERENCES}

1. Reimer K, Vogt PM, Broegmann B, et al. An innovative topical drug formulation for wound healing and infection treatment: in vitro and in vivo investigations of a povidone-iodine liposome hydrogel. Dermatology. 2000;201(3):235-41.

2. Unal S, Ersoz G, Demirkan F, et al. Analysis of skin-graft loss due to infection: infection-related graft loss. Ann Plast Surg. 2005;55(1):102-6.

3. Vogt PM, Hauser J, Rossbach O, et al. Polyvinyl pyrrolidone-iodine liposome hydrogel improves epithelialization by combining moisture and antisepis. A new concept in wound therapy. Wound Repair Regen. 2001;9(2):116-22. 
4. Leaper DJ, Durani P. Topical antimicrobial therapy of chronic wounds healing by secondary intention using iodine products. Int Wound J. 2008;5(2):361-8.

5. Edwards R, Harding KG. Bacteria and wound healing. Curr Opin Infect Dis. 2004;17(2):91-6.

6. Vehmeyer-Heeman M, Van den Kerckhove E, Gorissen $\mathrm{K}$, et al. Povidone-iodine ointment: no effect of split skin graft healing time. Burns. 2005;31(4):489-94.

7. Kramer A, Hübner NO, Weltmann KD, et al. Polypragmasia in the therapy of infected woundsconclusions drawn from the perspectives of low temperature plasma technology for plasma wound therapy. GMS Krankenhhyg Interdiszip. 2008;3(1):Doc13.

8. Leaper DJ, Schultz G, Carville K, et al. Extending the TIME concept: what have we learned in the past 10 years?(*). Int Wound J. 2012;9(Suppl 2):1-19.

9. van Meurs SJ, Gawlitta D, Heemstra KA, et al. Selection of an optimal antiseptic solution for intraoperative irrigation: an in vitro study. J Bone Jt Surg Am. 2014;96(4):285-91.

10. Reimer K, Fleischer W, Brögmann B, et al. Povidone-iodine liposomes-an overview. Dermatology. 1997;195(Suppl 2):93-9.

11. Martini L, Reali UM, Borgognoni, et al. Comparison of two dressing in the management of partial-thickness donor sites. J Wound Care. 1999;8(9):457-60.

12. Vogt PM, Reimer K, Hauser J, et al. PVP-Iodine in hydrosomes and hydrogel-a novel concept in wound therapy leads to enhanced epithelialization and reduced loss of skin grafts. Burns. 2006;32(6):698-705.

13. Vermeulen $\mathrm{H}$, Westerbos SJ, Ubbink DT. Benefit and harm of iodine in wound care: a systematic review. J Hosp Infect. 2010;76(3):191-9.

14. Muangman P, Nitimonton S, Aramwit P. Comparative clinical study of Bactigras and Telfa AMD for skin graft donor-site dressing. Int $\mathrm{J}$ Mol Sci. 2011;12:5031-8.

15. Eisenbeiß W, Siemers F, Amtsberg G, et al. Prospective, double-blinded, randomised controlled trial assessing the effect of an octenidine-based hydrogel on bacterial colonisation and epithelialization of skin graft wounds in burn patients. Int J Burns Trauma. 2012;2(2):71-9.

16. Silver GM, Robertson SW, Halerz MM, et al. A silver-coated antimicrobial barrier dressing used postoperatively on meshed autografts: a dressing comparison study. J Burn Care Res. 2007;28(5):715-9.

17. Assadian O, Arnoldo B, Purdue G, et al. A prospective, randomised study of a novel transforming methacrylate dressing compared with a silver-containing sodium carboxymethylcellulose dressing on partial-thickness skin graft donor sites in burn patients. Int Wound J. 2015;12(3):351-6.

18. Saleh K, Sonesson A, Persson K, et al. Can dressings soaked with polyhexanide reduce bacterial loads in full-thickness skin grafting? A randomized controlled trial. J Am Acad Dermatol. 2016;75(6):1221-8.

19. Gruber RP, Vistnes L, Pardoe R. The effect of commonly used antiseptics on wound healing. Plast Reconstr Surg. 1975;55(4):472-6. 